\title{
On description of magnetic stochasticity in poloidal divertor tokamaks
}

S. S. Abdullaev, M. Jakubowski, M. Lehnen, O. Schmitz, and B. Unterberg

Citation: Physics of Plasmas 15, 042508 (2008); doi: 10.1063/1.2907163

View online: https://doi.org/10.1063/1.2907163

View Table of Contents: http://aip.scitation.org/toc/php/15/4

Published by the American Institute of Physics

\section{Articles you may be interested in}

Non-linear magnetohydrodynamic modeling of plasma response to resonant magnetic perturbations Physics of Plasmas 20, 102510 (2013); 10.1063/1.4824820

Asymptotical and mapping methods in study of ergodic divertor magnetic field in a toroidal system

Physics of Plasmas 6, 153 (1999); 10.1063/1.873270

JET polari-interferometer

Review of Scientific Instruments 60, 2825 (1989); 10.1063/1.1140666

Divertor plasma detachment

Physics of Plasmas 23, 055602 (2016); 10.1063/1.4948273

Model of a magnetic field in poloidal divertor tokamaks affected by resonant magnetic perturbations

Physics of Plasmas 16, 030701 (2009); 10.1063/1.3083293

Modeling of stochastic magnetic flux loss from the edge of a poloidally diverted tokamak

Physics of Plasmas 9, 4957 (2002); 10.1063/1.1521125

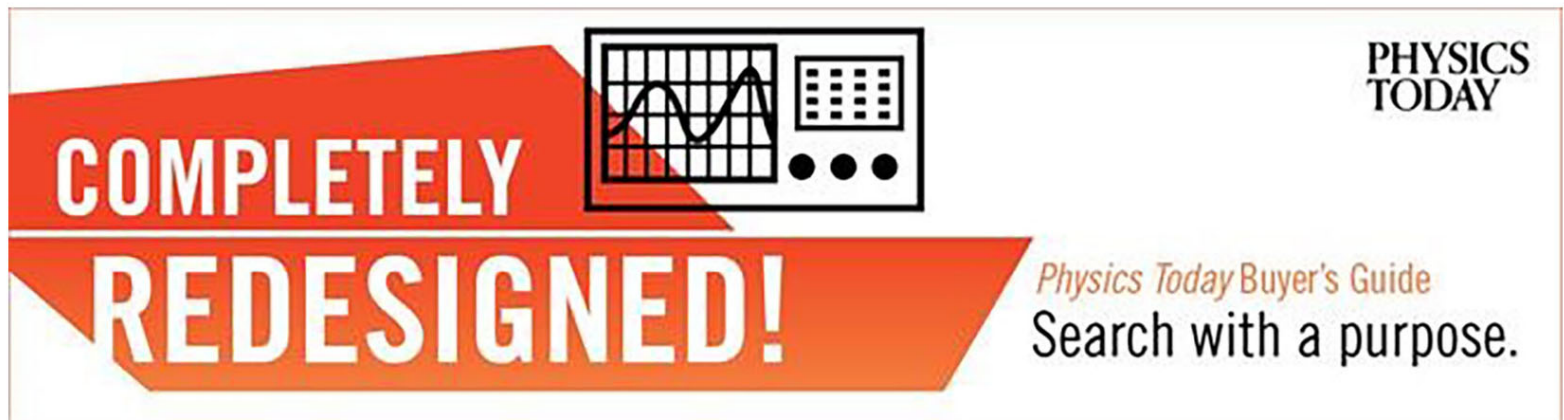




\title{
On description of magnetic stochasticity in poloidal divertor tokamaks
}

\author{
S. S. Abdullaev, M. Jakubowski, M. Lehnen, O. Schmitz, and B. Unterberg \\ Institut für Energieforschung-Plasmaphysik, Forschungszentrum Jülich GmbH, EURATOM Association, \\ Trilateral Euregio Cluster, D-52425 Jülich, Germany
}

(Received 8 January 2008; accepted 18 March 2008; published online 30 April 2008)

\begin{abstract}
A generic approach to study the stochastic field lines formed near the magnetic separatrix of poloidal divertor tokamaks due to nonaxisymmetric magnetic perturbations is proposed. The method is based on the determination of the so-called Poincare integral [S. S. Abdullaev, Phys. Rev. E 70, 046202 (2004)] defined as an integral over the vector potential of the perturbation field taken along the closed field lines orbit. This integral allows us to obtain the analytical estimations for the characteristics of chaotic field lines near the magnetic separatrix, like the Chirikov parameter, the widths of the stochastic layer and magnetic footprints, also the statistical characteristics of chaotic field lines, the quasilinear field line diffusion coefficients, and the Kolmogorov lengths. These estimations are in good agreement with the direct numerical calculations of corresponding quantities. A field line convection coefficient is introduced to describe the preferential outward drift of open chaotic field lines near the separatrix. (C) 2008 American Institute of Physics.
\end{abstract}

[DOI: $10.1063 / 1.2907163$ ]

\section{INTRODUCTION}

Recent encouraging experimental investigations on suppression of the edge localized modes (ELMs) in tokamaks, Doublet III D (DIII-D), and Joint European Torus (JET) (see, for example, Refs. 1-5) by applied external resonant magnetic perturbations have intensified the study of magnetic stochasticity in poloidal divertor tokamaks as given in Refs. 6-21. In ideal axisymmetric poloidal divertor tokamaks the magnetic separatrix sharply separates inner field lines of the confined plasma region from open field lines of the scapeoff-layer (SOL). Any small nonaxisymmetric magnetic perturbations split the magnetic separatrix into a pair of stable and unstable manifold intersections of which result in complex chaotic behavior of field lines (see, for example, Refs. 22 and 23).

The study of magnetic stochasticity in fusion devices goes back to the 1960s (see, e.g., Ref. 24, and references therein). The stochastic behavior of magnetic field lines near the magnetic separatrix due to its splitting has been, perhaps, first found by Melnikov (see Refs. 25 and 26). Destruction of magnetic surfaces near the stellator separatrix and the divertor separatrix, and the properties of the stochastic layer have been studied in Refs. 27-35.

In the early studies of magnetic stochasticity near the separatrix analytical models of the plasma had been used, and the criteria of stochasticity and the width of the stochastic layer has been found on the qualitative (Chirikov) criteria of overlapping of magnetic islands. The present studies of this problem given in Refs. 7-15, 17, and 19-21 are mostly based on the numerical field line tracing codes for the realistic magnetic configurations of the equilibrium plasma and the external perturbation coils. Although these approaches allow one to consider specific fusion devices, for instance, DIII-D or JET, it would be useful to an approach which reveals the generic features of the stochastic field lines near the separatrix.
In the present paper we intend to describe the universal features of chaotic field lines near the magnetic separatrix of poloidal divertor tokamaks. Since magnetic field lines are equivalent to the $1+1 / 2$ degrees-of-freedom Hamiltonian systems, we will apply for our approach the general results of the dynamics of Hamiltonian systems near the separatrix (see, e.g., Refs. 24, 36, and 37, and references therein). Based on these results, in particular, in Refs. 6, 38, and 39, we show that the properties of perturbed field lines near the separatrix are mostly determined by one quantity. This quantity is the generalized Poincare integral $R_{n}(h)$ defined as the integral from the toroidal component of the vector potential corresponding to the toroidal mode $n$ taken along closed unperturbed field lines.

The determination of only one function $R_{n}(h)$ (for a given toroidal mode $n$ ) is sufficient to estimate the main characteristics of field lines in a stochastic layer near the separatrix: the Chirikov parameter, the width of the stochastic layer, the quasilinear diffusion coefficients. They also allow us to estimate the Kolmogorov lengths, and the width of magnetic footprints on the divertor plates. It is important that in contrast to the conventional approaches developed in Refs. $7-10,12-14,17,19$, and 20 one does not require the calculation of the whole poloidal spectrum of modes $H_{m n}$ to estimate these quantities.

As was shown in numerous publications (see, e.g., Refs. 40-44) the description of open chaotic field lines at the plasma edge only by the diffusion coefficients of field lines is not complete, since the transport of particles and heat along these field lines becomes more convective, rather than diffusive. To quantify this property of open field lines we introduce the so-called field line convection coefficient, $u_{\mathrm{FL}}$. The latter describes the average outward drift of open field lines, and it gives the contribution $V_{\perp}=v_{s} u_{\mathrm{FL}}$ to the convection velocity ${ }^{53}$ in transport equations, where $v_{s}$ is a sound velocity. 


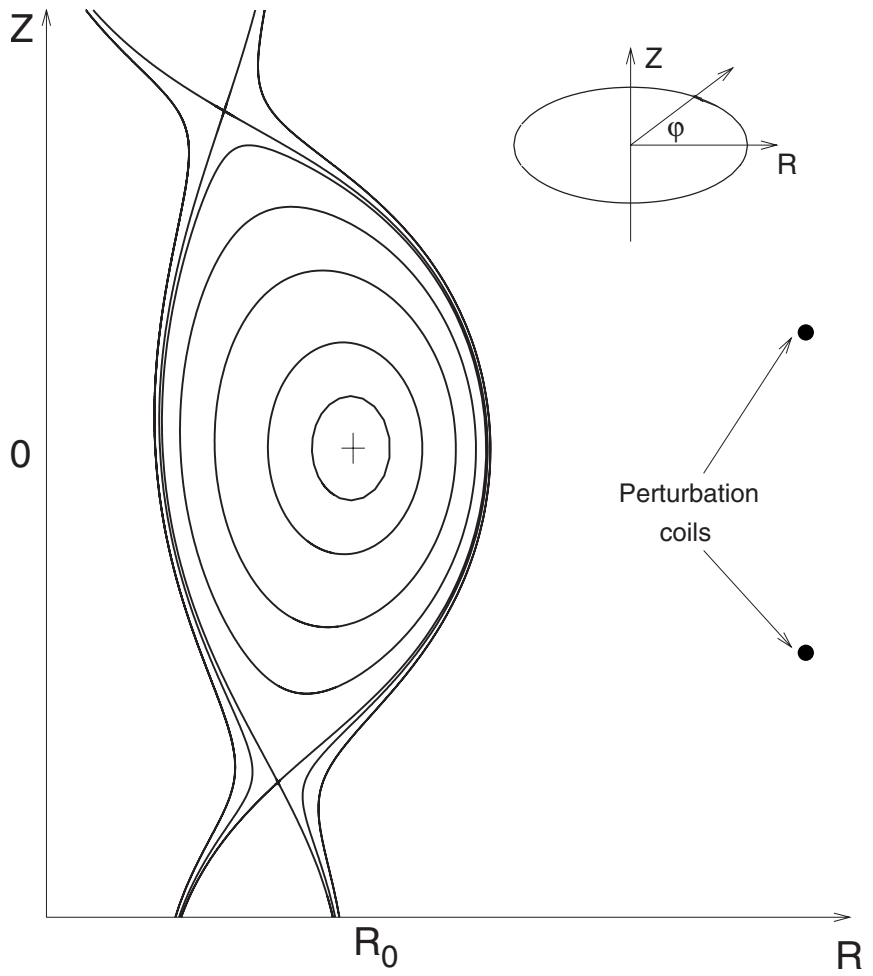

FIG. 1. Schematic view of poloidal divertor tokamak in the presence of external coil perturbation.

The present work is organized as follows: In Sec. II the main magnetic field line equations in Hamiltonian form and some general features of field lines near the magnetic separatrix of poloidal divertor tokamaks are recalled. Particularly, the field line equations in the presence of the nonaxisymmetric magnetic perturbations are also presented. The Poincaré integrals describing the effect of magnetic perturbations are introduced and their general properties are studied in Sec. III. There we also recall the so-called separatrix mapping which describes field lines near the magnetic separatrix. The properties of the stochastic layer of field lines near the separatrix are described in Sec. IV. Particularly, we obtain the estimations for the widths of the stochastic layer, magnetic footprints, field lines diffusion coefficients, the field line convection coefficients, and the Kolmogorov lengths. Conclusions are given in Sec. V. The calculation of the Kolmogorov length using the separatrix mapping is described in the Appendix.

\section{BASIC EQUATIONS}

We consider a single-null divertor tokamak under the influence of the nonaxisymmetric magnetic field perturbations of any origin, particularly created by external coils. The scheme of the problem is shown in Fig. 1. We suppose that the perturbation field penetrates into the edge plasma in a vacuum approximation without a plasma feedback.

\section{A. Field line equations}

Let $(R, Z, \varphi)$ be a cylindrical coordinate system where $R$ is directed along the major radius, $Z$ is the vertical coordi-

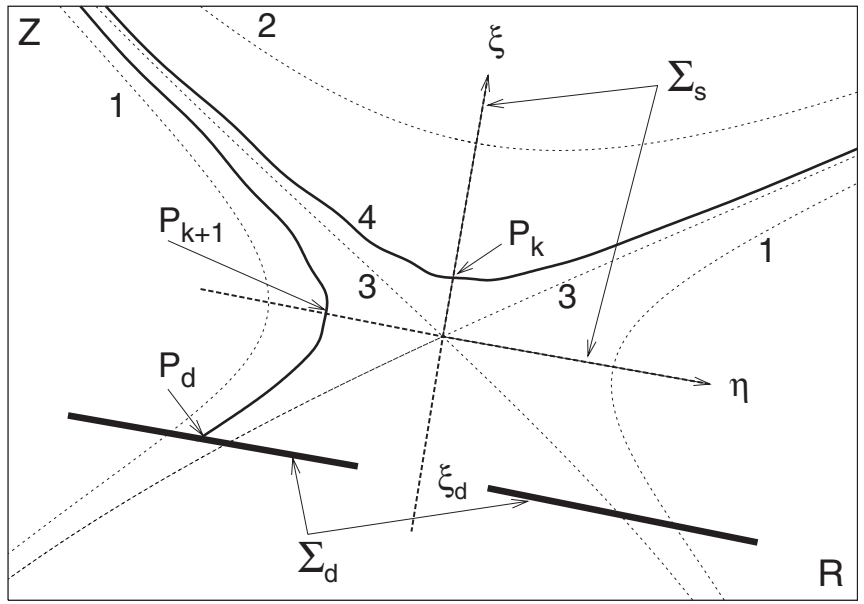

FIG. 2. Schematic view of magnetic field lines near the X-point and the separatrix mapping (18). Dotted curves 1-3 describe the unperturbed closed (curves 1), open (curve 2) field lines, and the separatrix (curve 3); solid curve 4 represents perturbed field lines; the section $\Sigma_{s}$ consists of two segments of the $\xi$ - and $\eta$-axes; the segments $\Sigma_{d}$ correspond to the divertor plates; $P_{k}(k=0, \pm 1, \pm 2, \ldots)$ are the sequence of crossing points of the perturbed field line with the $\xi$-and $\eta$-axes; $P_{d}$ is a hitting point of the perturbed field line with the divertor plate $\Sigma_{d} ; \xi_{d}$ is a $\xi$-coordinate of the divertor plates. The unperturbed field lines (curves 1 and 2 ) tranversely cross the axes $(\xi, \eta)$.

nate, and $\varphi$ is the toroidal angle (see Fig. 1). In this coordinate system the equations for field lines can be presented in the Hamiltonian form (see, for example, Refs. 6 and 45)

$$
\frac{d z}{d \varphi}=\frac{\partial H}{\partial p_{z}}, \quad \frac{d p_{z}}{d \varphi}=-\frac{\partial H}{\partial z} .
$$

The canonical variables $\left(z, p_{z}\right)$ are related to the coordinates $(R, Z, \varphi): z=Z / R_{0}, p_{z}=\ln \left(R / R_{0}\right)$, where $R_{0}$ is the major radius of the torus center. The toroidal angle $\varphi$ plays the role of a timelike independent variable, and the poloidal flux represents the Hamiltonian function $H$ which is determined by the normalized $\varphi$-component of the vector potential

$$
H \equiv H\left(z, p_{z}, \varphi\right)=\frac{R A_{\varphi}}{B_{0} R_{0}^{2}} .
$$

The poloidal magnetic field components, $B_{R}, B_{z}$, are determined by $B_{R}=-\partial A_{\varphi} / \partial Z=-\left(B_{0} R_{0}^{2} / R^{2}\right) \partial H / \partial z$, $B_{z} R^{-1} \partial\left(R A_{\varphi}\right) / \partial R=\left(B_{0} R_{0}^{2} / R^{2}\right) \partial H / \partial p_{z}$.

In the axisymmetric case $A_{\varphi}=A_{\varphi}(R, Z)$. The magnetic surfaces are determined by contour lines of $R A_{\varphi}(R, Z)$ $=$ const. The toroidal flux $\psi$, normalized to $R_{0}^{2} B_{0}$, as a function of the poloidal flux for the given magnetic surface can be found as a closed integral

$$
\psi(H)=(2 \pi)^{-1} \oint_{C} p_{z}(z, H) d z,
$$

taken along the closed contour $C$ of $H=H_{0}\left(z, p_{z}\right)=$ const. The safety factor $q(H)$ is then given by $q(\psi)=d \psi / d H$.

Consider the field lines near the $\mathrm{X}$-point which are schematically shown in Fig. 2. Let $\left(z=z_{s}, p_{z}=p_{s}\right)$ be the coordinate of the X-point in which the null of the poloidal magnetic field is the first order. The latter means that the expansion of the poloidal magnetic field $\left(B_{R}, B_{Z}\right)$ near this point contains 
nonzero coefficients before the first order terms, i.e., $B_{R}, B_{Z}$ $\approx a_{z}\left(z-z_{s}\right)+a_{p}\left(p_{z}-p_{s}\right)$, where $a_{z} \neq 0, a_{p} \neq 0$. Then by the linear transformation of coordinates $\left(z, p_{z}\right)$ to the coordinates $(\xi, \eta)$

$$
\begin{aligned}
& \xi=\left(z-z_{s}\right) \cos \alpha_{0}+\left(p_{z}-p_{s}\right) \sin \alpha_{0}, \\
& \eta=-\left(z-z_{s}\right) \sin \alpha_{0}+\left(p_{z}-p_{s}\right) \cos \alpha_{0},
\end{aligned}
$$

where $\alpha_{0}$ is the angle between the $z$ axis and the $\xi$ axis, the Hamiltonian near the X-point can be presented in a normal form,

$$
H=H_{0}(0,0)-\frac{\alpha^{2}}{2} \xi^{2}+\frac{\beta^{2}}{2} \eta^{2}
$$

where $\alpha$ and $\beta$ are positive constants. Unperturbed field lines transversely cross the axis $(\xi, \eta)$. The poloidal magnetic field $\left(B_{R}, B_{Z}\right)$ near the $\mathrm{X}$-point are linear functions $\xi$, $\eta$, i.e., $B_{R}, B_{Z} \sim a_{1} \xi+a_{2} \eta$ with nonzero constant coefficients $a_{1}, a_{2}$.

Near the separatrix the safety factor has universal asymptotics

$$
q(h)=\frac{1}{2 \pi \gamma} \ln \frac{Q}{|h|}+\mathcal{O}(h)
$$

where $h=H-H_{0}(0,0)$ is the relative poloidal flux, $\gamma=\alpha \beta$, $Q>0$ are a positive constant depending on the specific magnetic field configuration. As seen from Eq. (6) the safety factor near the separatrix is mainly determined by the parameter $\gamma$, while the dependence on $Q$ is weak. Note that $h$ is the relative poloidal flux. At the separatrix $h=0$; it is negative for the field lines inside the plasma region $(h<0)$, positive for the open field lines $(h>0)$.

Consider also the second order null of the poloidal magnetic field at the X-point $\left(z=z_{s}, p_{z}=p_{s}\right)$ when the coefficients $\alpha, \beta$ in the expansion of $H$ near the X-point given by Eq. (5) (or $a_{1}, a_{2}$ in the expansion of $B_{R}, B_{Z}$ ) vanish, and the next nonvanishing expansion coefficients in Hamiltonian $H$ are in terms of the order of $O\left(\xi^{3}\right)$, and $B_{R}, B_{Z} \sim a_{11} \xi^{2}+a_{12} \xi \eta$ $+a_{22} \eta^{2}$. This kind of divertor tokamaks with the second order of null of the poloidal magnetic field have been recently proposed in Ref. 46 in order to expand the flux of heat load over a much wider area than in the case of the standard divertor discussed above. The expansion of the Hamiltonian near the points $\left(z=z_{s}, p_{z}=p_{s}\right)$ then contain the third order terms, and can be presented as

$$
h=-A\left(\xi^{3}-3 \xi \eta^{2}\right)+B\left(3 \xi^{3} \eta-\eta^{3}\right),
$$

where $A$ and $B$ are constants. For simplicity, we suppose that $B=0$. Then one can show that the asymptotics of the safety factor near the separatrix has the form

$$
q(h)=\frac{C}{|h|^{s}}+a h+b h^{2}+\cdots, \quad h \rightarrow 0,
$$

where $C$ and $s$ are positive constants independent of $h$, i.e., $C>0, s>0$, and $a, b$ are constants.

\section{B. Magnetic perturbation}

Consider the effect of nonaxisymmetric magnetic perturbations. The magnetic perturbations can be described by the vector potential

$$
A_{\varphi}^{(\mathrm{per})}(R, Z, \varphi)=\epsilon \frac{B_{0} R_{0}^{2}}{R} \sum_{n} A_{n}(R, Z) \cos \left(n \varphi+\chi_{n}\right),
$$

where $n$ stands for the toroidal mode number, the dimensionless perturbation parameter, $\epsilon$, is introduced as $\epsilon$ $=\max \left|A_{\varphi}^{(\mathrm{per})}\right| / B_{0} R_{0}$, and $\chi_{n}$ is the phase of the toroidal mode $n$. For the model of the plasma considered in Ref. 6 the parameter $\epsilon$ is equal to $\epsilon=I_{c} / I_{p}$.

Then according to Eq. (2) the perturbation Hamiltonian $\epsilon H_{1}\left(z, p_{z}, \varphi\right)$ can be written as

$$
H_{1}\left(z, p_{z}, \varphi\right)=\sum_{n} H_{n}\left(z, p_{z}\right) \cos \left(n \varphi+\chi_{n}\right),
$$

where $H_{n}\left(z, p_{z}\right)=A_{n}\left[R\left(p_{z}\right), R_{0} z\right]$.

The effect of any nonaxisymmetric perturbation can also be studied by presenting the equations (1) of field lines in magnetic coordinates, i.e., toroidal flux, $\psi$, and poloidal angle, $\vartheta$,

$$
\frac{d \vartheta}{d \varphi}=\frac{\partial H}{\partial \psi}, \quad \frac{d \psi}{d \varphi}=-\frac{\partial H}{\partial \vartheta},
$$

with the Hamiltonian function written in the form

$$
\begin{aligned}
& H(\psi, \vartheta, \varphi)=\int \frac{d \psi}{q(\psi)}+\epsilon H_{1}(\psi, \vartheta, \varphi), \\
& H_{1}(\psi, \vartheta, \varphi)=\sum_{m n} H_{m n}(\psi) \cos \left(m \vartheta-n \varphi+\chi_{m n}\right) .
\end{aligned}
$$

The strength of resonant magnetic perturbations which destroys the magnetic surfaces $\psi_{m n}, q\left(\psi_{m n}\right)=m / n$, is given by the Fourier components $H_{m n}(\psi)$, resulting in the formation of magnetic islands chain.

Furthermore the main statements will be illustrated for the three-wire plasma model of the plasma described in Ref. 6 . The parameters of the model are chosen close to the ones of the JET: the major radius $R_{0}=3 \mathrm{~m}$, the toroidal field $B_{0}$ $=2 \mathrm{~T}$, the plasma current $I_{p}=2 \mathrm{MA}$. The magnetic perturbations are created by $N$ pair of loop coils with opposite flowing currents $\pm I_{c}$ (see Fig. 1, and Fig. 7 in Ref. 6). For this model we have the values of the parameters $\alpha=0.713, \beta$ $=0.620, \gamma=0.442$. A numerical integration of field line equations are performed using the accurate mapping method described in Refs. 6 and 24, unless it is noted.

\section{POINCARÉ-TYPE INTEGRALS}

The so-called Poincaré integrals play an important role in the dynamics of motion near the separatrix (see, e.g., Ref. 22). The generalization of these integrals has been introduced in Refs. 24, 38, and 39 for construction of canonical mappings for description of motion near the separatrix. The latter, $R_{n}(h)$, is defined as the integral, 


$$
\begin{aligned}
R_{n}(h) & =K_{n}(h)+i L_{n}(h) \\
& =\int_{-\pi q(h)}^{\pi q(h)} H_{n}\left(z(h, \varphi), p_{z}(h, \varphi)\right) e^{i n \varphi} d \varphi,
\end{aligned}
$$

taken over the functions $H_{n}\left(z, p_{z}\right)$ along the closed field line $z(h, \varphi), p_{z}(h, \varphi)$. At the separatrix $h=0$ this integral coincides with the Poincare integral as given in Ref. 22. The integrals $R_{n}(h)$ can be presented as a sum of regular, $R_{n}^{(\mathrm{reg})}(h)$, and oscillatory, $R_{n}^{\text {(osc) }}(h)$, parts, i.e., see Refs. 24 and 39,

$$
R_{n}(h)=R_{n}^{(\mathrm{reg})}(h)+R_{n}^{(\mathrm{osc})}(h),
$$

where $R_{n}^{(\mathrm{reg})}(h)$ represents the smoothly varying part, and $R_{n}^{(\text {osc })}(h)$ describes the rapidly oscillating part. Near the separatrix the leading term of the asymptotical expansion of $R_{n}^{(\text {osc })}(h)$ in series of powers $|h|$ is described by the formula type,

$$
R_{n}^{\text {(osc) }}(h) \approx A|h|^{1 / 2} \sin (\pi n q(h))+O(h),
$$

where the constant parameter $A$ is not dependent on $h$. The $R_{n}^{\text {(osc) }}(h)$ has zeros at the primary resonance values of $h_{m n}$, $q\left(h_{m n}\right)=m / n: R_{n}^{(\text {osc })}\left(h_{m n}\right)=0$. The amplitude of oscillations $R_{n}^{\text {(osc) }}(h)$ also ceases when approaching the separatrix $h \rightarrow 0$ as $R_{n}^{\text {(osc) }}(h) \propto|h|^{1 / 2}$.

A typical dependence of the $R_{n}(h)$ on the relative poloidal flux $h$ for the toroidal mode $n=1$ is shown in Fig. 3(a): solid curve 1 describes the integral $R_{n}(h)$ itself, dashed curve 2, the regular part $R_{n}^{(\mathrm{reg})}(h)$. As seen from Fig. 3(a) $R_{n}(h)$ is oscillating around $R_{n}^{\text {(reg) }}(h)$. Figure $3(\mathrm{~b})$ shows the dependence of $R_{n}(0)$ on the toroidal mode number $n$.

Near the separatrix and at the separatrix $h=0$ the integrals $R_{n}^{(\mathrm{reg})}(h)$ decay exponentially with the toroidal mode number $n$. Analytical and numerical calculations show that $R_{n}(0) \propto \exp (-C n / \gamma)$, where $C$ is a constant parameter independent of $\gamma$.

There exists the important relation between the Fourier coefficients $H_{m n}(\psi)$ in Eq. (12) taken at the corresponding resonant magnetic surfaces, $\psi_{m n}$, and the Poincaré integrals $R_{n}(h)$ (see Ref. 39),

$$
H_{m n}\left(\psi_{m n}\right)=\frac{1}{2 \pi q\left(\psi_{m n}\right)} R_{n}^{*}\left(h_{m n}\right),
$$

where $h_{m n}=h\left(\psi_{m n}\right)$. This relation allows us to write the width of the magnetic island through only one function, $R_{n}^{(\mathrm{reg})}(h)$, for a given toroidal mode number $n$,

$W_{m n}=4 q\left(\psi_{m n}\right)\left|\frac{\epsilon H_{m n}\left(\psi_{m n}\right)}{d q / d \psi}\right|^{1 / 2}=4\left|\frac{\epsilon q\left(\psi_{m n}\right) R_{n}\left(h_{m n}\right)}{2 \pi d q / d \psi}\right|^{1 / 2}$.

Therefore, instead of evaluation of the spectrum of perturbation, $H_{m n}$, for all poloidal mode numbers $m$ at the given toroidal mode $n$, it is sufficient to determine only one function, $R_{n}^{(\mathrm{reg})}(h)$.
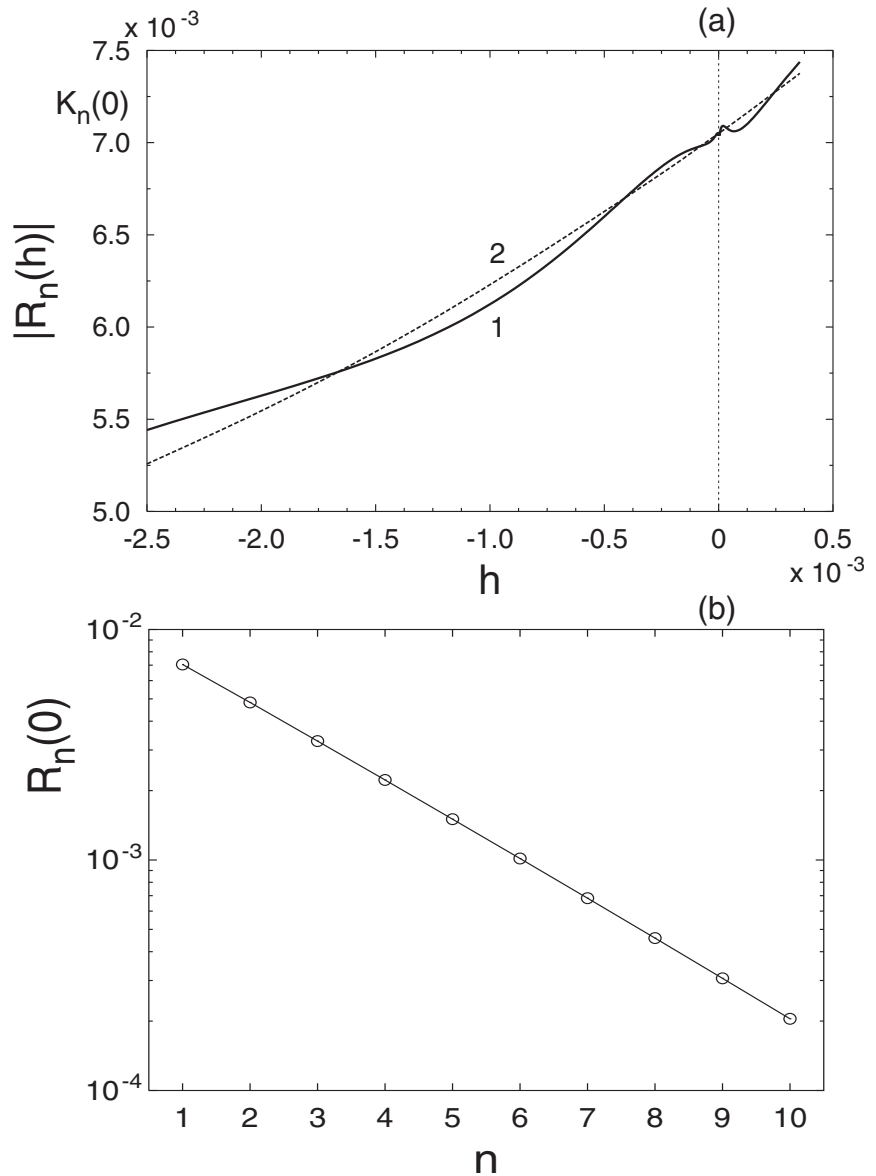

FIG. 3. (a) Typical dependence of the integral $R_{n}(h)$ on the relative poloidal flux $h$ for the toroidal mode $n=1$ : curve 1 describes the function $R_{n}(h)$; curve 2 describes its regular part $R_{n}^{(\mathrm{reg})}(h)$. (b) Exponential dependence of $R_{n}(0)$ on the toroidal mode $n$ at the separatrix $h=0$.

\section{A. Separatrix mapping}

The separatrix mapping is a very efficient tool to study field lines near the separatrix and X-point. It allows one to obtain the qualitative and quantitative characteristics of chaotic field lines such as the formation of a stochastic layer in the plasma region and the structure of magnetic footprints on the divertor plate (see Refs. 6 and 24).

The scheme of the separatrix mapping is shown in Fig. 2. Let $P_{k}$ be the $k$ th crossing point of a perturbed field line with the section $\Sigma_{s}$ consisting of segments along the axes $\xi, \eta$. The separatrix mapping relates the point $P_{k}$ with the next crossing point $P_{k+1}, P_{k+1}=\hat{M} P_{k}$. For the full description one should introduce a forward and backward mapping, which maps the field line along the positive and negative direction of the toroidal axis $\varphi$, respectively. The crossing point $P_{k}$ is defined by the canonical variables: the toroidal angle, $\varphi_{k}$, and the relative poloidal flux, $h_{k}$. The simplified form of the separatrix map for the single-null divertor tokamak is given by

$$
h_{k+1}=h_{k} \mp \epsilon \sum_{n} n\left[K_{n}(0) \sin \Phi_{n}^{(\mp)}+L_{n}(0) \cos \Phi_{n}^{(\mp)}\right] \text {, }
$$




$$
\varphi_{k+1}=\varphi_{k} \pm \pi\left[q\left(h_{k}\right)+q\left(h_{k+1}\right)\right] \text {, }
$$

where $\Phi_{n}^{(\mp)}=n\left[\varphi_{k} \pm \pi q\left(h_{k}\right)\right]+\chi_{n}$. The upper (lower) signs correspond to the forward (backward) mapping.

The following rules are applied to the mapping given by Eq. (18). It is supposed that a field line is inside the plasma region when the relative poloidal flux $h$ is negative at the crossing point of a field line with the upper branch of the $\xi$-axis, $h_{k}<0$. If $h_{k}>0$ it is supposed that the field line crosses the $\eta$-axis and then hits the divertor plate $\Sigma_{d}$ (see Fig. 2). This assumption works practically well for the sufficiently small values of the perturbation parameter $\epsilon$, which is confirmed with the numerical computations in Refs. 39.

To determine magnetic footprints on divertor plates $\Sigma_{d}$ one needs to map field lines from the sections $\Sigma_{s}$ to the sections $\Sigma_{d}$. Since these sections are located close to the $X$-point these field lines can be traced along unperturbed field lines starting from its coordinates $P_{k}=\left(\varphi_{k}, h_{k}\right)$ at $\Sigma_{s}$ until they reach the divertor plate $\Sigma_{d}$ at the point $P_{d}=\left(\varphi_{d}, h_{d}\right)$ (see Fig. 2 ). The toroidal angle, $\varphi_{d}$, and the relative poloidal flux, $h_{d}$, on the divertor plate are determined as

$$
h_{d}=h_{k}, \quad \varphi_{d}=\varphi_{k}+\Delta \varphi\left(h_{k}\right),
$$

where $\Delta \varphi(h)$ is the increment of the toroidal angle $\varphi$ necessary to reach the plate $\Sigma_{d}$ along unperturbed field lines on the magnetic surface of constant $h=h\left(z, p_{z}\right)$.

\section{THE PROPERTIES OF THE STOCHASTIC LAYER}

\section{A. The generic structure}

It is known that any small nonaxisymmetric perturbations of Eq. (9) destroy the magnetic separatrix forming the region of chaotic field lines near the unperturbed separatrix (see, for example, Refs. 23 and 36). These field lines are not confined anymore in the plasma region, and they leave the plasma region hitting the divertor plates after a certain number of toroidal (or poloidal) turns.

Actually the structure of the stochastic layer near the separatrix is not uniformly chaotic, but it consists also regions of regular field lines, the so-called KolmogorovArnold-Moser (KAM) stability islands (see, e.g., Refs. 24 and 47). It is most convenient to present the structure of the stochastic layer in the $(\varphi, \psi)$ and $(\vartheta, \psi)$ planes. Particularly, the presentation in the $(\varphi, \psi)$ allows one to elevate the toroidal mode content. A typical stochastic layer created by a magnetic perturbation with toroidal mode $n=3$ is shown in Figs. 4: (a) in the $(\vartheta, \psi)$ plane; (b) in the $(\varphi, \psi)$ plane; (c) the Chirikov overlapping parameter $\sigma$ [see its definition below given by Eq. (20)] versus the toroidal flux $\psi$.

As seen from Figs. 4(a) and 4(b) one can distinguish between three different regions of the stochastic layer. The first outer region, $\psi_{f}<\psi<\psi_{a}$, adjacent to the unperturbed magnetic separatrix consists of long stripes, the so-called fingers, along which field lines from the inner region of the plasma leave the system. In the inner region, $\psi_{s}<\psi<\psi_{f}$, where the Chirikov parameter of overlapping neighboring islands $\sigma$ is of order 1, the chaotic field lines fill the region more uniformly, except tiny size KAM islands. The lower part of the stochastic layer, $\psi_{c}<\psi<\psi_{s}$, bordering with the (a)

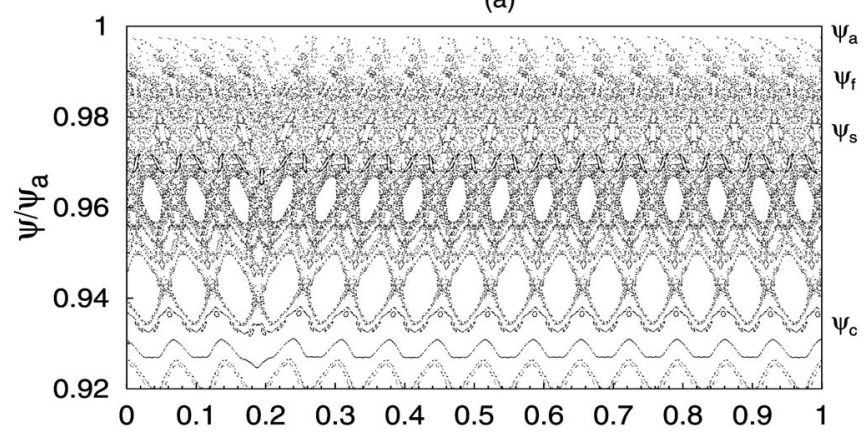

(b) $\vartheta / 2 \pi$
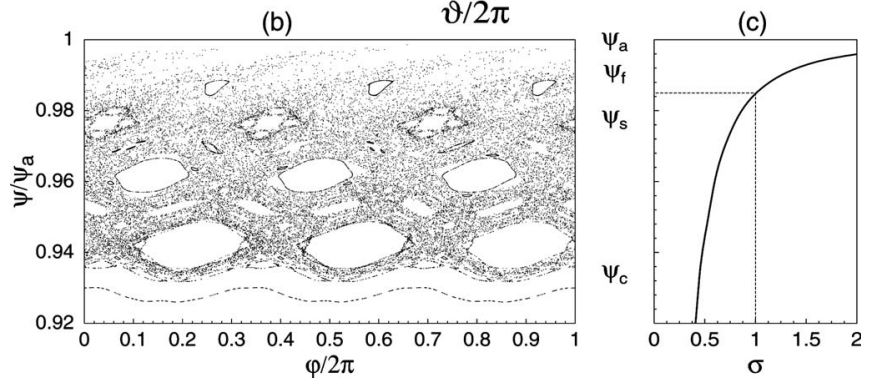

FIG. 4. Poincaré sections of field lines near the separatrix in the $(\vartheta, \psi)$ plane (a) and $(\varphi, \psi)$ plane (b). (c) Chirikov parameter $\sigma$ vs the normalized flux $\psi$. The perturbation parameter $\epsilon=0.01$.

last intact magnetic surface, consists of KAM stability islands. The sizes of the latter increases towards smaller $\psi$.

The outermost region of field lines bordering with the unperturbed separatrix can be conveniently presented by the so-called laminar plot, i.e., a contour plot of lengths of field lines connecting the inner divertor plate with the outer divertor plate. Such a plot is shown in Fig. 5 where the connection lengths are measured in a number of poloidal turns. The minimal number of poloidal turns of field lines entering into the plasma region is equal to 2 shown in the figure by dark blue areas. As seen from Fig. 5 the field lines with a large number of connection lengths may go deeper into the plasma along three pairs of stripes.

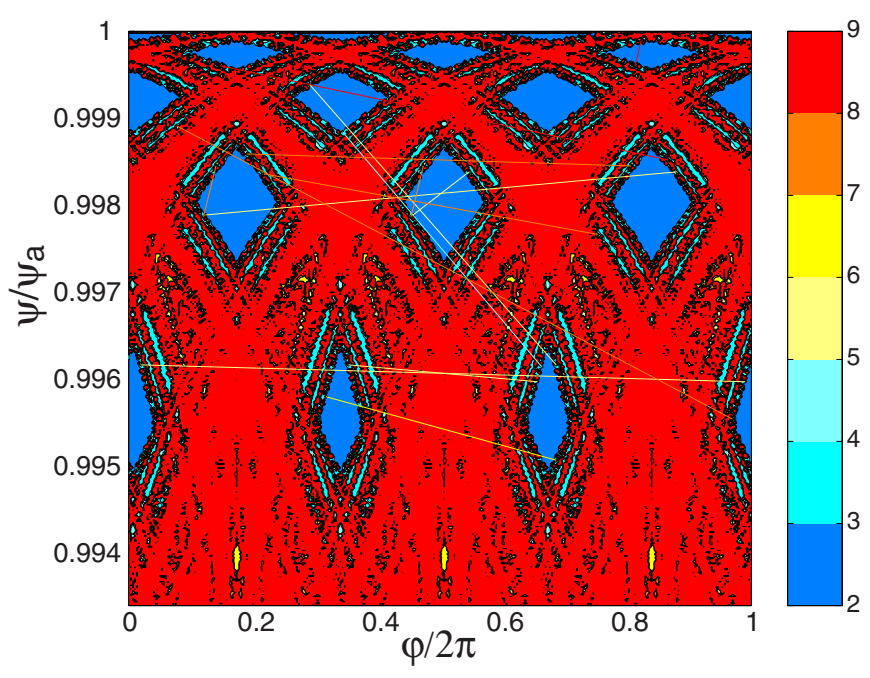

FIG. 5. (Color online) Contour plot of wall to wall connection lengths of field lines in the $(\varphi, \psi)$ plane, obtained by the separatrix mapping (18). The corresponding Poincaré sections were shown in Figs. 4(a) and 4(b). 
Field lines in the laminar zone are predominantly directed outward. This gives rise to the nondiffusive (convective) contribution to the heat and particle transport from the inner regions of the plasma. Such a transport has been found and extensively investigated in the Dynamic Ergodic Divertor (DED) of the Tokamak Experiment for Technology Oriented Research (TEXTOR) tokamak (see, for example, Refs. 42-44). See below for a quantitative description of this transport where a field line convection coefficient is introduced (see Sec. IV D).

\section{B. Width of the stochastic layer}

To estimate the width of the stochastic layer usually the Chirikov's criteria of overlapping of neighboring magnetic islands is used. As we see below (see also Ref. 4) this approach does not give an estimation for the width of the entire stochastic layer, but for its highly ergodized outer part bordering with the unperturbed separatrix as shown in Figs. 4(b) and 4(c).

To estimate the width of the stochastic layer we use the relative poloidal flux $h$ instead of the toroidal flux $\psi$. Resonant magnetic surfaces, $h_{m n}$, defined by $q\left(h_{m n}\right)=m / n$, near the separatrix become dense, i.e., the distance between neighboring resonant magnetic surfaces, $\delta h=\left|h_{m+1, n}-h_{m n}\right|$, goes asymptotically to zero with increasing the poloidal mode number $m$, or equivalently with decreasing $|h|$. Using the asymptotics of the safety factor near the separatrix given in Eq. (6) and the width of the $(m, n)$ magnetic island in the $h$ variable, $\Delta h=W_{m n} / q\left(h_{m n}\right)$, one obtains the Chirikov criteria of overlapping of neighboring islands

$$
\sigma^{2}(h)=\left(\frac{\Delta h}{\delta h}\right)^{2}=\frac{4 n^{2} \epsilon\left|R_{n}(h)\right|}{\pi^{2} \gamma|h|} \gtrsim 1 .
$$

The condition Eq. (20) is satisfied for the motion near the separatrix with $|h|<h_{c}$, where $h_{c}$ is the boundary value of the stochastic layer determined by the equation, $\sigma\left(h_{c}\right) \sim 1$,

$$
\left|h_{c}\right| \approx \frac{4 n^{2} \epsilon\left|R_{n}(0)\right|}{\pi^{2} \gamma} .
$$

The width of the stochastic layer is linearly proportional to the perturbation parameter $\left|h_{c}\right| \propto \epsilon$. Using the relation Eq. (5) one can obtain the width of the stochastic layer along the spatial coordinate $X=R_{0} \xi: X_{c}=\sqrt{2\left|h_{c}\right|} / \alpha \propto \epsilon^{1 / 2}$. As seen from Figs. 4(b) and 4(c) the condition $\sigma \gtrsim 1$ is satisfied only in the outermost region of the stochastic layer.

Consider the case of the poloidal divertor with the second order null of the poloidal field mentioned at the end of Sec. II A. Using the asymptotics of the safety factor in this case given by Eq. (6) one can obtain the following estimation for the width of the stochastic layer:

$$
\left|h_{c}\right| \approx\left(\frac{8 s n^{2} \epsilon\left|R_{n}(0)\right|}{\pi C}\right)^{1 /(1+s)} \propto \epsilon^{1 /(1+s)} .
$$

Using Eq. (7) we have the estimation for the spatial width of the stochastic layer $X_{c} \approx\left(\left|h_{c}\right| / A\right)^{1 / 3} \propto \epsilon^{1 / 3(1+s)}$. Therefore, the stochastic layer formed near the separatrix due to nonaxisymmetric magnetic perturbations in these types of poloidal divertor tokamaks would have significantly larger extension

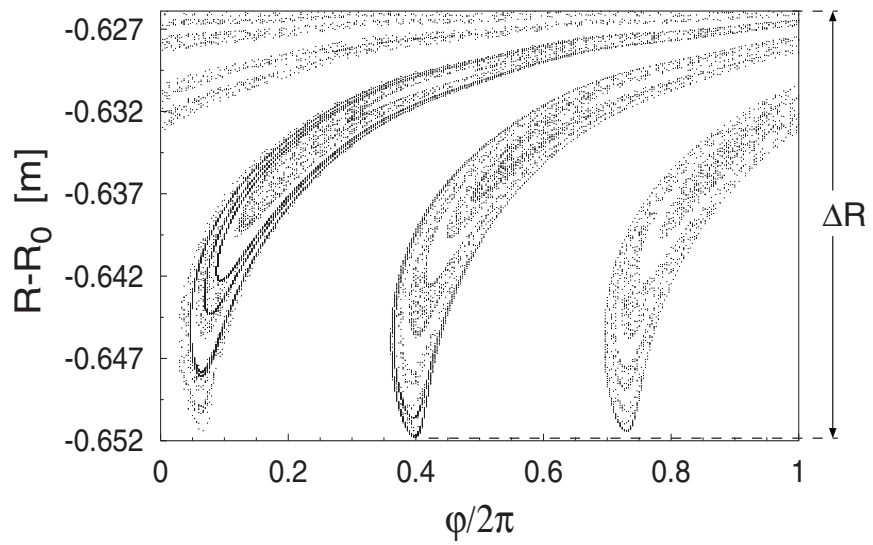

FIG. 6. Magnetic footprints on the inner divertor plate. $\Delta R$ is a width of the magnetic footprint along the major radial coordinate $R$. The divertor plate is located at the distance $\left|X_{d}\right|=\left|R_{0} \xi_{d}\right|=0.2 \mathrm{~m}$ from the X-point along the $\xi$-axis (see Fig. 2). The perturbation parameter $\epsilon=0.05$.

than for the standard X-point divertor tokamaks with the first order null of the poloidal field at the X-point.

\section{Width of magnetic footprints}

The heat and particle deposition patterns on the divertor plate are closely related to the magnetic footprints, i.e., the hitting points of open field lines with the divertor plates (see, for example, Ref. 11). The typical pattern of a magnetic footprint obtained by the Runge-Kutta integration scheme of field line equations is shown in Fig. 6 in the $(\varphi, R)$ plane on the inner divertor plate. As seen there, it consists of three spiral-like stripes.

The radial extension of magnetic footprints can be analytically estimated using the separatrix mapping given by Eq. (18). A field line with the initial coordinates $\left(\varphi_{0}, h_{0}\right),\left(h_{0}\right.$ $<0)$ in the plasma region leaves the plasma when it crosses the axis $\eta$, i.e., $h_{k-1}<0$ and $h_{k}>0$ (see Fig. 2). According to the first equation in Eq. (18) the maximal value of the poloidal flux $h_{k}$ carried by these field lines can reach the value

$$
h_{\max } \leqslant \epsilon n R_{n}(0), \quad R_{n}(0)=\sqrt{K_{n}^{2}(0)+L_{n}^{2}(0)} .
$$

This estimation is in good agreement with the direct numerical calculations of the magnetic footprints. For example, for the perturbation parameter $\epsilon=0.05$ from the numerical calculation one has $h_{\max } \approx 3.5 \times 10^{-4}$ which is close to $h_{\max } \approx 4.2$ $\times 10^{-4}$ obtained by the mapping. The numerical calculations show also a linear dependence of $h_{\max }$ on the perturbation parameter $\epsilon$. The latter is plotted in Fig. 7: curve 1 describes the $h_{\max }$ versus $\epsilon$, curve 2 corresponds to the fitted linear function $h=a \epsilon$ with $a=6.92069 \times 10^{-3}$.

We estimate the radial width $\Delta R$ for the simple divertor geometry shown in Fig. 2. Using the relation $R=R_{0} \exp \left(p_{z}\right)$ and the expansion given Eq. (5) for the case when the divertor plates are sufficiently close to the X-point one can obtain 


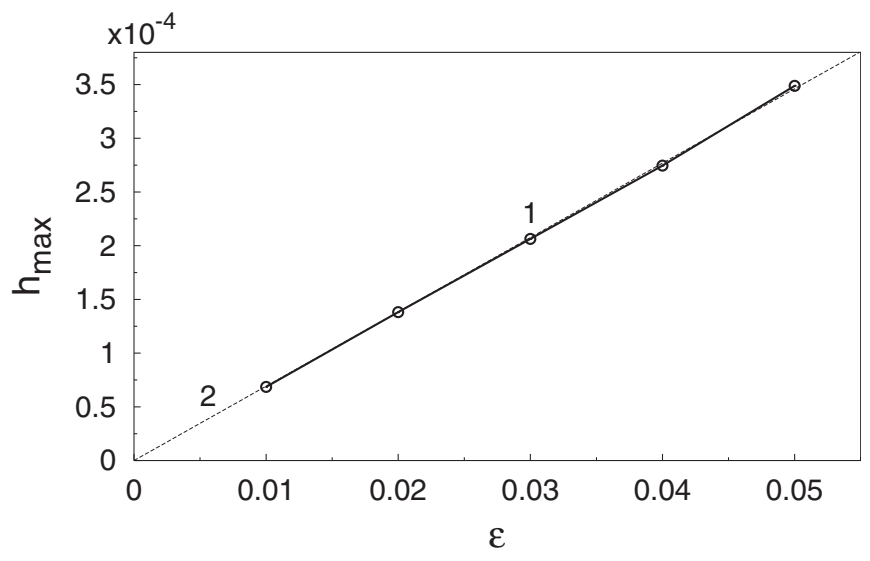

FIG. 7. Dependence of the $h_{\max }$ on the perturbation parameter $\epsilon$ obtained by the numerical integration: curve 1 describes the $h_{\max }$ vs $\epsilon$, dashed curve 2 corresponds to the fitted linear function $h=a \epsilon$ with $a=6.9 \times 10^{-3}$.

$$
\Delta R=R_{0}\left\{\exp \left[p_{z}\left(h_{\max }, \xi_{d}\right)\right]-\exp \left[p_{z}\left(0, \xi_{d}\right)\right]\right\},
$$

where $p_{z}(h, \xi)=p_{s}+\cos \alpha_{0} \sqrt{2} / \beta \sqrt{h+\alpha^{2} X_{d}^{2} / 2 R_{0}^{2}}, X_{d}=R_{0} \xi_{d}$ is the $\xi$-coordinate of the divertor plate (see Fig. 2). For $h_{\text {max }} R_{0} /\left|\alpha X_{d}\right| \ll 1$, we have

$$
\Delta R \approx \frac{\cos \alpha_{0} h_{\max } R_{0}^{2}}{\sqrt{2}\left|X_{d}\right| \gamma} \leqslant \frac{\epsilon n R_{n}(0)}{\gamma} \cos \alpha_{0} \frac{R_{0}^{2}}{\sqrt{2} X_{d}} .
$$

Using the values $\gamma=0.442, R_{0}=3 \mathrm{~m},\left|X_{d}\right|=0.2 \mathrm{~m}$ for our model of the plasma according to Eq. (24) we have the approximate value $\Delta R \approx 3.1 \mathrm{~cm}$ for the radial width of magnetic footprint for the perturbation parameters $\epsilon=0.05$, which is close the value $\Delta R=2.6 \mathrm{~cm}$ obtained from the numerical calculations (see Fig. 6). Usually the width of the magnetic footprints does not exceed the estimation given by Eqs. (24) and (25).

\section{Diffusion and "convection" of field lines}

To study the transport of particles and heat in the stochastic magnetic field one requires the estimation of field line diffusion coefficients and the Kolmogorov lengths (see, e.g., Ref. 48). Below we derive an quasilinear formula for the field line diffusion coefficients near the magnetic separatrix.

For the Hamiltonian system given Eqs. (11) and (12), the quasilinear diffusion coefficient $D_{\psi}(h)=\left\langle(\Delta \psi)^{2}\right\rangle / 2 \Delta \varphi$ along the toroidal flux coordinate, $\psi$, is given by

$$
D_{\psi}(h)=\frac{\pi \epsilon^{2}}{2} \sum_{m}\left|m H_{m n}(\psi)\right|^{2} \delta\left[\frac{m}{q(\psi)}-n\right] .
$$

The diffusion coefficient of field lines, $D_{\mathrm{FL}}=\left\langle(\Delta S)^{2}\right\rangle / 2 \Delta l$, along the spatial coordinate $(S)$ normal to the magnetic surface, $\psi(R, Z)=$ const, can be expressed through $D_{\psi}(h)$. To be specific consider the diffusion along the $X=R_{0} \xi$ axis (see Fig. 2). Then near the separatrix according to Eq. (5) we have $\Delta \psi=(d \psi / d h) \Delta h=R_{0}^{-1} q(h) \alpha|h|^{1 / 2} \Delta X$. On the other hand, the element of the field line length is equal to $\Delta l=R_{0} \Delta \varphi$. Using these relations and Eqs. (16) and (26), we have

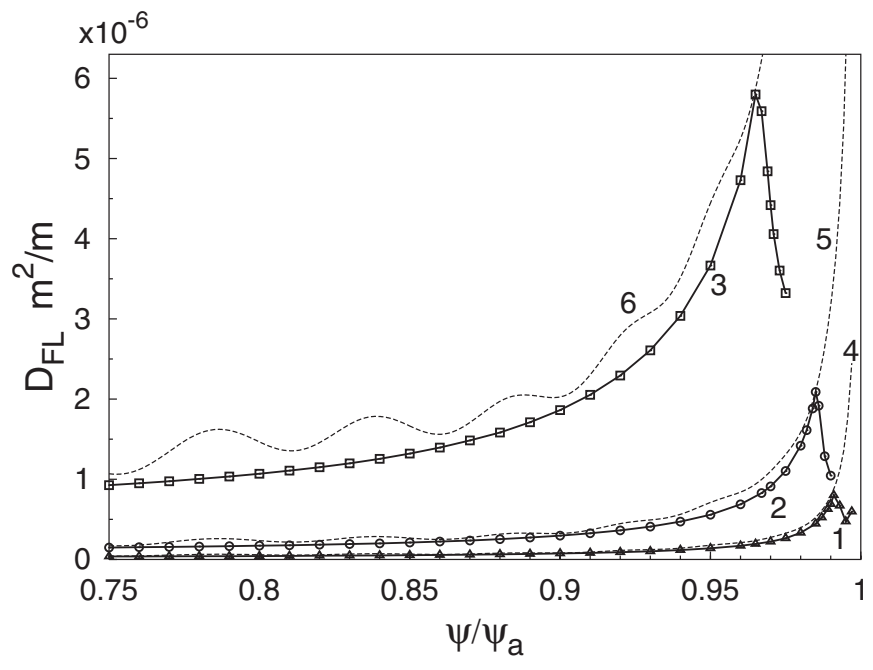

FIG. 8. Field line diffusion coefficients at the different amplitudes of perturbations calculated numerically (curves 1-3) and according to the quasilinear formula (27) (curves 4-6): curves 1 and 4 correspond to $\epsilon=0.01$; curves 2 and 5 correspond to $\epsilon=0.02$; curves 3 and 6 correspond to $\epsilon$ $=0.05$.

$$
D_{\mathrm{FL}}(h)=\frac{R_{0} \epsilon^{2} n^{2}}{16 \pi \alpha^{2} q\left(h_{m n}\right)|h|}\left|R_{n}\left(h_{m n}\right)\right|^{2} .
$$

For the validation of this quasilinear formula we have also directly calculated the field line diffusion coefficients $D_{\text {FL }}$ using the procedure described in Refs. 24, 49, and 51.

The dependencies of $D_{\mathrm{FL}}$ on the toroidal magnetic flux obtained by the numerical calculations and the quasilinear formula are plotted in Fig. 8 for the three different values of the perturbation parameter $\epsilon$ : curves 1 and 4 correspond to $\epsilon=0.01$, curves 2 and $5-\epsilon=0.02$, curves 3 and $6-\epsilon=0.05$. The numerical diffusion coefficients are described by curves 1-3, the quasilinear formulas, by curves 4-6. For example, the magnetic field line diffusivity $D_{\mathrm{FL}}$ for the perturbation parameter $\epsilon=0.05$ takes the value $3.66 \times 10^{-6} \mathrm{~m}$ at the normalized toroidal flux $\psi / \psi_{a}=0.95$. For the plasma discharges in the ELMs mitigation experiments by the resonant magnetic perturbation in DIII-D reported in Ref. 1 the sound velocity is $v_{s}=5.5 \times 10^{5} \mathrm{~m} / \mathrm{s}$. The corresponding stochastic particle diffusivity $\chi_{p}=v_{s} D_{\mathrm{FL}}$ has the value $2.01 \mathrm{~m}^{2} / \mathrm{s}$, which is of order of the anomalous particle diffusivity.

As seen from Fig. 8 the quasilinear values of the diffusion coefficients $D_{\mathrm{FL}}$ closely follow the numerical values, although they slightly exceed the numerical one. The latter fact is due to the remnants of islands in the stochastic layer (see Fig. 4).

However, in the laminar zone where stochastic field lines have a short connection lengths the transport of field lines cannot be described as a diffusive process similar to the laminar zone in the ergodic divertors (see Refs. 42-44 and 49). Therefore the quasilinear formula is not valid in this region (see Fig. 8).

In order to describe the transport of field lines in the laminar zone we introduce statistical characteristics of chaotic field lines related to the convection velocity in the transport equations. For simplicity we consider low-collisional plasmas. Let $v_{\perp}$ be a component of a test particle velocity 


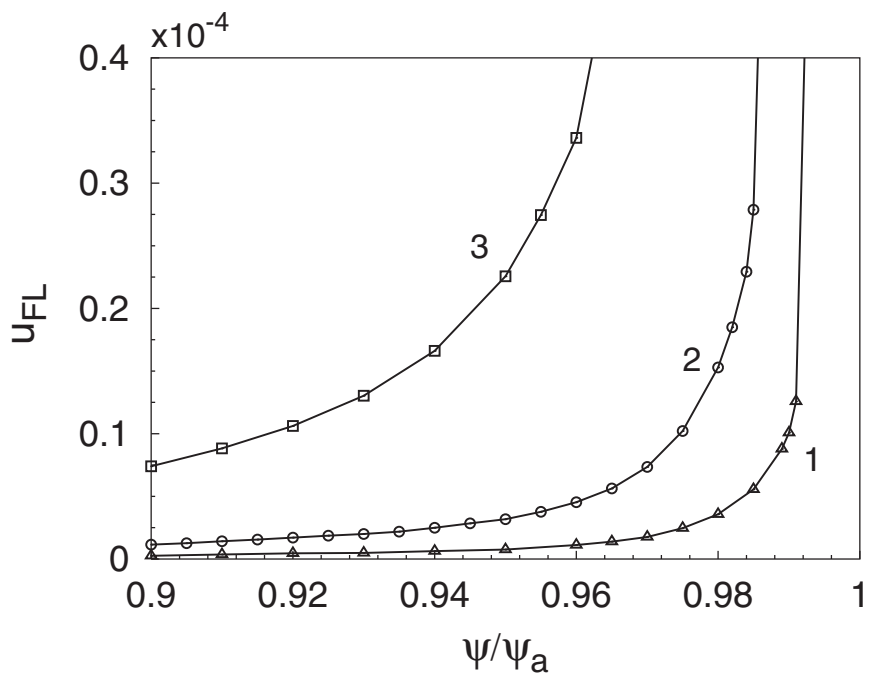

FIG. 9. Field line "pinch" coefficients, $u_{\mathrm{FL}}$, at the different amplitudes of perturbations: curve 1, $\epsilon=0.01$; curve $2, \epsilon=0.02$; curve $3, \epsilon=0.05$.

directed along the coordinate $X$ normal to the magnetic surfaces. It can be presented as $v_{\perp}=d X(t) / d t=(d l / d t)$ $\times[d X(t) / d l]=v_{\|}[d X(t) / d l]$, where $v_{\|}$is a velocity of the parallel motion of the particle, $d l$ is a length element on the field line. The averaging of $v_{\perp}$ over the magnetic surface gives the pinch velocity $V_{\perp}=v_{\|} u_{\mathrm{FL}}$, where the quantity

$$
u_{\mathrm{FL}}=\left.\left\langle\frac{d X(l)}{d l}\right\rangle\right|_{\psi=\text { const }},
$$

describes the average drift of field lines in the stochastic layer, and it can be called a field line convection coefficient. The averaging $\langle\cdots\rangle$ is performed over field lines on the given magnetic surface $\psi$.

In the case of highly ergodized stochastic zones with the long wall to wall connection lengths one expects that the coefficient $u_{\mathrm{FL}}$ is negligibly small since field lines equally cross the unperturbed magnetic surface in both the inward and outward directions. However, in the laminar zone where field lines have the short connection lengths the convection coefficient $u_{\mathrm{FL}}$ may have reached significant values since particles following the open field lines which left the plasma region are terminated on the divertor plates and do not come back to the plasma region. Such an outward drift of chaotic open field lines may contribute significantly to the convectional outward transport of heat and particles at the plasma edge.

Figure 9 shows the radial profiles of the field line convection coefficients, $u_{\mathrm{FL}}$, in the stochastic layer formed at the three different perturbation coefficients: $\epsilon=0.01$ (curve 1), 0.02 (curve 2), and 0.05 (curve 3 ). The value of $u_{\mathrm{FL}}$ grows radially when it approaches the unperturbed separatrix. For instance, at the perturbation amplitude $\epsilon=0.05$ it reaches the value $u_{\mathrm{FL}}=3 \times 10^{-5}$ at the normalized toroidal flux $\psi / \psi_{a}$ $=0.95$. The sound velocity is $v_{s}=5.5 \times 10^{5} \mathrm{~m} / \mathrm{s}$ and the corresponding convection velocity $V_{\perp}=v_{s} u_{\mathrm{FL}}$ has the value $\sim 16.5 \mathrm{~m} / \mathrm{s}$, which is of the order of the anomalous convection velocity in tokamaks (see, for example Ref. 50, p. 196).

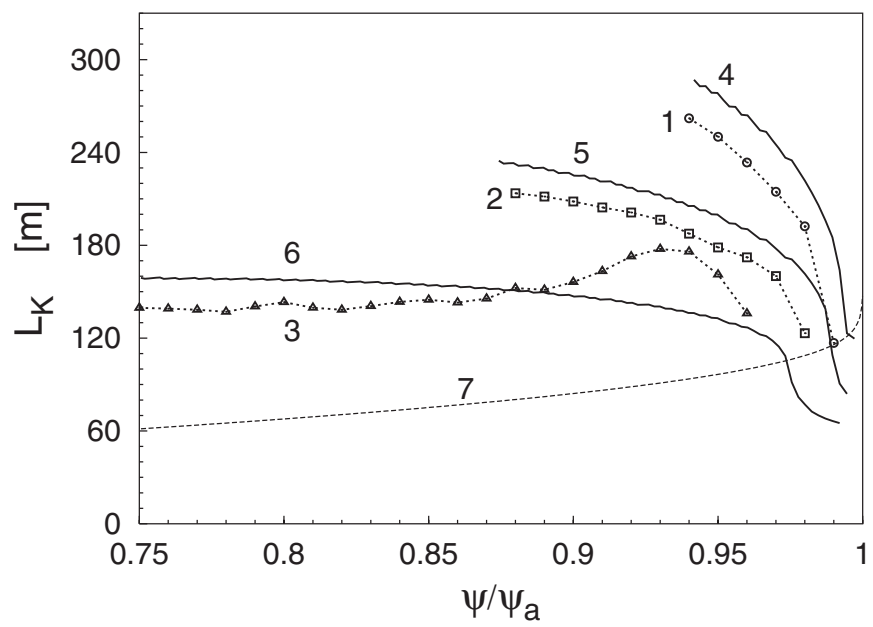

FIG. 10. Kolmogorov lengths of field lines at the different amplitudes of perturbations: curves 1 and 4 corresponds to $\epsilon=0.01$; curves 2 and 5 correspond to $\epsilon=0.02$; curves 3 and 6 correspond to $\epsilon=0.05$; curve 7 describes the connection length $L_{c}=2 \pi q R_{0}$. Curves 1-3 are obtained by integration of field lines Eqs. (11) and (12); curves $4-6$ are obtained by separatrix mapping (18).

\section{E. The Kolmogorov length}

The Kolmogorov length, $L_{K}$, is a decorrelation length of chaotic field lines. This statistical characteristics can be found by averaging the maximal Lyapunov exponents $\sigma$ of field lines in the stochastic layer. The latter characterizes the inverse of mean length of exponential divergency of chaotic field lines. The procedure of calculations of $\langle\sigma(\psi)\rangle$ using the mapping technique of integration of the Hamiltonian equations given by Eqs. (11) and (12), is described in Refs. 51 and 52. The Kolmogorov lengths are also determined using the separatrix mapping (18) which is described in the Appendix.

In Fig. 10 the profiles of the local Kolmogorov lengths are plotted for the three different values of the perturbation parameter $\epsilon$ : $\epsilon=0.01$ (curves 1 and 4), $\epsilon=0.02$ (curves 2 and 5), and $\epsilon=0.05$ (curves 3 and 6). Curves 1-3 are obtained using by the integration of field line equations (11) and (12); and curves $4-6$ are obtained by separatrix mapping given by Eq. (18). As seen from Fig. 10 the Kolmogorov length has an order of a connection length $L_{c}=2 \pi q(\psi) R_{0}$ (curve 7 in Fig. 10). Although the Kolmogorov lengths calculated by the separatrix mapping exceed the corresponding values calculated from the direct integration, both values have the same tendency; they monotonically decrease when one approaches the separatrix.

\section{CONCLUSIONS}

We have described a generic method to study the stochastic field lines formed near the magnetic separatrix of poloidal divertor tokamaks in the presence of nonaxisymmetric magnetic perturbations. The method is based on the introduction of the generalized Poincaré integrals, $R_{n}(h)$, defined as an integral taken over the toroidal component of the vector potential of the perturbation field along the closed magnetic fields. This integral alone (for a given toroidal mode $n$ ) is enough to calculate the quantitative characteris- 
tics of the stochastic layer of field lines, the Chirikov parameter, the widths of the stochastic layer, and the magnetic footprints on the divertor plate. It allowed one to estimate the statistical characteristics of chaotic field lines, quasilinear diffusion coefficients of field lines, and the Kolmogorov lengths. For these estimations one does not need the determination of the set of poloidal spectrum $H_{m n}$ of the magnetic field perturbations.

We have introduced new statistical characteristics of chaotic field lines near the separatrix, a field line convection coefficient, quantifying a preferential outward drift of open field lines. Together with the field line diffusion coefficients this quantity allows one to estimate the stochastic transport characteristics, the additional contributions to the diffusion and the convection velocity due to open chaotic field lines. It is shown that the convectional velocity of particles due to the outward drift of chaotic open field lines may reach the values of order of anomalous convection velocity.

\section{ACKNOWLEDGMENTS}

The authors are grateful to Dr. M. Tokar for useful discussions and comments.

This work has partially been supported by the German Research Foundation under Grant No. UN 265/1-1.

\section{APPENDIX: LYAPUNOV EXPONENTS}

Below we calculate the Lyapunov exponents and the corresponding Kolmogorov lengths using the separatrix mapping given by Eq. (18). Consider the Jacobi matrix,

$$
\hat{J}_{k}=\left(\begin{array}{cc}
\frac{\partial h_{k+1}}{\partial h_{k}} & \frac{\partial h_{k+1}}{\partial \varphi_{k}} \\
\frac{\partial \varphi_{k+1}}{\partial h_{k}} & \frac{\partial \varphi_{k+1}}{\partial \varphi_{k}}
\end{array}\right) .
$$

Since $\operatorname{det}|J|=1$, the eigenvalues of this matrix $\lambda_{1,2}$ are given by

$$
\lambda_{1,2}^{(k)}=\frac{1}{2}\left(\operatorname{Tr} \hat{J}_{k} \pm \sqrt{\operatorname{Tr} \hat{J}_{k}-2}\right) .
$$

From the separatrix map Eq. (18) follows that

$$
\begin{aligned}
\operatorname{Tr} \hat{J}_{k}= & 2+\pi \epsilon n^{2}\left(K_{n} \cos \Phi_{n}^{( \pm)}-L_{n} \sin \Phi_{n}^{( \pm)}\right) \\
& \times\left[\frac{\partial q\left(h_{k}\right)}{\partial h_{k}}+\frac{\partial q\left(h_{k+1}\right)}{\partial h_{k+1}}\right] .
\end{aligned}
$$

The finite time (or finite step $N$ ) Lyapunov exponent is defined as (see, e.g., Ref. 51),

$$
\sigma_{N}=\frac{1}{N} \ln \prod_{k=1}^{N} \lambda^{(k)}
$$

where $\lambda^{(k)}$ is the largest eigenvalue, i.e., $\lambda^{(k)}=\max \left(\lambda_{1}^{(k)}, \lambda_{2}^{(k)}\right)$. The local Kolmogorov length, $L_{K}$ is determined as an inverse of averaged $\bar{\sigma}_{N}$,

$$
L_{K}(\psi)=\frac{L_{c}}{\left\langle\sigma_{N}\right\rangle}, \quad\left\langle\sigma_{N}\right\rangle=\frac{1}{M} \sum_{i=1}^{M} \sigma_{N}(i),
$$

where $L_{c}=2 \pi q(\psi) R_{0}$ is the length of a field line over one poloidal turn, $\bar{\sigma}_{N}$ is the averaged value of $\sigma_{N}$ taken over all $M$ field lines with initial coordinates on the magnetic surface with a given $\psi$.

${ }^{1}$ T. E. Evans, R. A. Moyer, K. H. Burrel, M. E. Fenstermacher, I. Joseph, A. W. Leonard, T. H. Osborne, G. D. Porter, M. J. Schaffer, P. Snyder, P. Thomas, J. G. Watkins, and W. P. West, Nat. Phys. 2, 419 (2006).

${ }^{2}$ T. E. Evans, R. A. Moyer, P. R. Thomas, J. G. Watkins, T. H. Osborne, J. A. Boedo, E. J. Doyle, M. E. Fenstermacher, K. H. Finken, R. J. Groebner, M. Groth, J. H. Harris, R. J. LaHaye, C. J. Lasnier, S. Masuzaki, N. Ohyabu, D. G. Pretty, T. L. Rhodes, H. Reimerdes, D. L. Rudakov, M. J. Schaffer, G. Wang, and L. Zeng, Phys. Rev. Lett. 92, 235003 (2004).

${ }^{3}$ T. E. Evans, R. A. Moyer, J. G. Watkins, T. H. Osborne, P. R. Thomas, M. Becoulet, J. A. Boedo, E. J. Doyle, M. E. Fenstermacher, K. H. Finken, R. J. Groebner, M. Groth, J. H. Harris, G. L. Jackson, R. J. La Haye, C. J. Lasnier, S. Masuzaki, N. Ohyabu, D. G. Pretty, H. Reimerdes, T. L. Rhodes, D. L. Rudakov, M. J. Schaffer, M. R. Wade, G. Wang, W. P. West, and L. Zeng, Nucl. Fusion 45, 595 (2005).

${ }^{4}$ Y. Liang, H. R. Koslowski, P. R. Thomas, E. Nardon, B. Alper, P. Andrew, Y. Andrew, G. Arnoux, Y. Baranov, M. Bécoulet, M. Beurskens, T. Biewer, M. Bigi, K. Crombe, P. de Vries, W. Fundamenski, S. Gerasimov, C. Giroud, M. P. Gryaznevich, N. Hawkes, S. Hotchin, D. Howell, S. Jachmich, V. Kiptily, L. Moreira, V. Parail, S. D. Pinches, E. Rachlew, and O. Zimmermann, Phys. Rev. Lett. 98, 265004 (2007).

${ }^{5}$ R. A. Moyer, T. Evans, T. Osborne, P. R. Thomas, M. Becoulet, J. Harris, K. H. Finken, J. A. Boedo, E. J. Doyle, M. E. Fenstermacher, P. Gohil, R. J. Groebner, M. Groth, G. L. Jackson, R. J. La Haye, C. J. Lasnier, A. W. Leonard, G. R. McKee, H. Reimerdes, T. L. Rhodes, D. L. Rudakov, M. J. Schaffer, P. B. Snyder, M. R. Wade, G. Wang, J. G. Watkins, W. P. West, and L. Zeng, Phys. Plasmas 12, 056119 (2005).

${ }^{6}$ S. S. Abdullaev, K. H. Finken, M. Jakubowski, and M. Lehnen, Nucl. Fusion 46, S113 (2006).

${ }^{7}$ M. Bécoulet, G. Huysmans, P. Thomas, P. Ghendrih, E. Nardon, A. Grosman, X. Garbet, W. Zwingmann, R. A. Moyer, T. E. Evans, M. Schaffer, and A. Leonard, Nucl. Fusion 45, 1284 (2005).

${ }^{8}$ M. Bécoulet, E. Nardon, G. Huysmans, P. Thomas, M. Lipa, W. Zwingmann et al., Proceedings of the 21st International Conference on Fusion Energy 2006, Ghengdi, 2006 (IAEA, Vienna, 2006), pp. IT/P--29.

${ }^{9}$ M. Bécoulet, E. Nardon, P. Thomas, G. Huysmans, F. Dubois, J. F. Artaud, F. Imbeaux, M. Lipa, P. Ghendrih, A. Grosman, R. A. Moyer, T. E. Evans, M. Schaffer, V. Chuyanov, and G. Federici, Proceedings of the 32nd EPS Conference on Plasma Physics, Tarragona, 2005, Europhysics Conference Abstracts 29C (European Physical Society, Mulhouse, 2005), pp. 2.005.

${ }^{10}$ T. E. Evans, K. H. Burrel, M. E. Fenstermacher, R. A. Moyer, T. H. Osborne, M. J. Schaffer, W. P. West, L. W. Yan, J. A. Boedo, E. J. Doyle, G. L. Jackson, I. Joseph, C. J. Lasnier, A. W. Leonard, T. L. Rhodes, P. Thomas, J. G. Watkins, and L. Zeng, Phys. Plasmas 13, 056121 (2006).

${ }^{11}$ T. E. Evans, I. Joseph, R. A. Moyer, M. E. Fenstermacher, C. J. Lasnier, and L. W. Yan, J. Nucl. Mater. 363-365, 570 (2007).

${ }^{12}$ T. E. Evans, R. A. Moyer, and P. Monat, Phys. Plasmas 9, 4957 (2002).

${ }^{13}$ T. E. Evans, R. K. Roeder, J. A. Carter, and B. I. Rapoport, Contrib. Plasma Phys. 44, 235 (2004).

${ }^{14}$ T. E. Evans, R. K. Roeder, J. A. Carter, B. I. Rapoport, M. E. Fenstermacher, and C. J. Lasnier, J. Phys.: Conf. Ser. 7, 174 (2005).

${ }^{15}$ I. Joseph, R. A. Moyer, T. E. Evans, M. J. Schaffar, A. M. Runov, R. Schneider, S. V. Kasilov, M. Groth, and M. E. Fenstermacher, J. Nucl. Mater. 363-365, 591 (2007).

${ }^{16}$ Longwen Yan, T. E. Evans, S. M. Kaye, and R. Maingi, Nucl. Fusion 46, 858 (2006).

${ }^{17}$ E. Nardon, M. Bécoulet, G. Huysmans, O. Czarny, P. Thomas, M. Lipa, R. A. Moyer, T. E. Evans, G. Federici, Y. Gribov, A. Polevoi, G. Saibene, A. Portone, and A. Loarte, J. Nucl. Mater. 363-365, 1071 (2007).

${ }^{18}$ A. Punjabi, H. Ali, T. Evans, and A. H. Boozer, Phys. Plasmas 14, 082503 (2007).

${ }^{19}$ R. K. W. Roeder, B. I. Rapoport, and T. Evans, Phys. Plasmas 10, 3796 (2003).

${ }^{20}$ M. J. Schaffer, J. E. Menard, M. P. Aldan, J. M. Bialek, T. E. Evans, and R. A. Moyer, Nucl. Fusion 48, 024004 (2007). 
${ }^{21}$ L. W. Yan and T. E. Evans, J. Nucl. Mater. 363-365, 723 (2007).

${ }^{22}$ V. I. Arnold, V. V. Kozlov, and A. I. Neishtadt, Mathematical Aspects of Classical and Celestial Mechanics, Encyclopedia of Mathematical Sciences. Dynamical Systems (Springer, Berlin, 1988), Vol. 3.

${ }^{23}$ A. J. Lichtenberg and M. A. Lieberman, Regular and Stochastic Motion (Springer, New York, 1992).

${ }^{24}$ S. S. Abdullaev, Construction of Mappings for Hamiltonian Systems and Their Applications, Lecture Notes in Physics (Springer-Verlag, Berlin Heidelberg, 2006), Vol. 691.

${ }^{25}$ V. K. Melnikov, Sov. Phys. Dokl. 7, 502 (1962).

${ }^{26}$ V. K. Melnikov, Sov. Phys. Dokl. 8, 362 (1963).

${ }^{27}$ S. S. Abdullaev and K. H. Finken, Nucl. Fusion 38, 531 (1998).

${ }^{28}$ S. S. Abdullaev and G. M. Zaslavsky, Phys. Plasmas 3, 516 (1996).

${ }^{29}$ A. H. Boozer and A. B. Rechester, Phys. Fluids 21, 682 (1978).

${ }^{30}$ R. J. La Haye, Nucl. Fusion 31, 1550 (1991).

${ }^{31}$ N. Pomphrey and A. Reiman, Phys. Fluids B 4, 938 (1992).

${ }^{32}$ A. Punjabi, A. Verma, and A. H. Boozer, Phys. Rev. Lett. 69, 3322 (1992).

${ }^{33}$ D. A. Skinner, T. H. Osborne, S. C. Prager, and W. Park, Phys. Fluids 30, 1218 (1987).

${ }^{34}$ Y. Tomita, H. Momota, and R. Itatani, J. Phys. Soc. Jpn. 44, 637 (1978).

${ }^{35}$ Y. Tomita, S. Seki, and H. Momota, J. Phys. Soc. Jpn. 42, 687 (1977).

${ }^{36}$ B. V. Chirikov, Phys. Rep. 52, 265 (1979).

${ }^{37}$ G. M. Zaslavsky, R. Z. Sagdeev, D. A. Usikov, and A. A. Chernikov, Weak Chaos and Quasiregular Patterns (Cambridge University Press, Cambridge, 1991).

${ }^{38}$ S. S. Abdullaev, Phys. Rev. E 70, 046202 (2004).

${ }^{39}$ S. S. Abdullaev, Phys. Rev. E 72, 046202 (2005).

${ }^{40}$ P. Ghendrih, A. Grossman, and H. Capes, Plasma Phys. Controlled Fusion 38, 1653 (1996).

${ }^{41}$ S. S. Abdullaev, K. H. Finken, M. Jakubowski, S. Kasilov, M. Kobayashi,
D. Reiser, D. Reiter, A. Runov, and R. Wolf, Nucl. Fusion 43, 299 (2003). ${ }^{42}$ M. W. Jakubowski, O. Schmitz, S. S. Abdullaev, S. Brezinsek, K. H. Finken, A. Krämer-Flecken, M. Lehnen, U. Samm, K. H. Spatschek, B. Unterberg, R. C. Wolf, and the TEXTOR Team, Phys. Rev. Lett. 96, 035004 (2006).

${ }^{43}$ O. Schmitz, D. Harting, S. S. Abdullaev, S. Brezinsek, K. H. Finken, H. Frerichs, M. Jakubowski, M. Lehnen, X. Loozen, P. Mertens, D. Reiter, U. Samm, B. Schweer, G. Sergienko, M. Tokar, B. Unterberg, R. Wolf, and the TEXTOR Team, J. Nucl. Mater. 363-365, 680 (2007).

${ }^{44}$ O. Schmitz, M. W. Jakubowski, H. Frerichs, D. Harting, M. Lehnen, B. Unterberg, S. S. Abdullaev, S. Brezinsek, I. Classen, T. Evans, Y. Feng, K. H. Finken, M. Kantor, D. Reiter, U. Samm, B. Schweer, G. Sergienko, M. Tokar, E. Uzgel, R. Wolf, and the TEXTOR Team, Nucl. Fusion 48, 024009 (2008).

${ }^{45}$ S. R. Barocio, E. Chávez-Alarcón, and D. Gutierrez-Tapia, Braz. J. Phys. 36, 550 (2006).

${ }^{46}$ D. D. Ryutov, Phys. Plasmas 14, 064502 (2007)

${ }^{47}$ S. S. Abdullaev, Phys. Rev. E 62, 3508 (2000).

${ }^{48}$ M. Z. Tokar, T. E. Evans, A. Gupta, R. Singh, P. Kaw, and R. C. Wolf, Phys. Rev. Lett. 98, 095001 (2007).

${ }^{49}$ S. S. Abdullaev, K. H. Finken, and K. H. Spatschek, Phys. Plasmas 6, 153 (1999).

${ }^{50}$ J. Wesson, Tokamaks, 3rd ed. (Clarendon, Oxford, 2004).

${ }^{51}$ K. H. Finken, S. S. Abdullaev, M. Jakubowski, M. Lehnen, A. Nicolai, and K. H. Spatschek, The Structure of Magnetic Field in the TEXTOR$D E D$, Energy Technology (Forschungszentrum Julich, Julich, Germany, 2005), Vol. 45, ISSN 1433-5522 (also available online at http://www.fzjuelich.de/zb/datapool/page/439/00312_Finken.pdf).

${ }^{52}$ S. S. Abdullaev, K. H. Finken, A. Kaleck, and K. H. Spatschek, Phys. Plasmas 5, 196 (1998).

${ }^{53}$ The convection velocity is also known as a pinch velocity. 